\title{
Real world effectiveness of warfarin among ischemic stroke patients with atrial fibrillation: observational analysis from Patient-Centered Research into Outcomes Stroke Patients Prefer and Effectiveness Research (PROSPER) study
}

\author{
Ying Xian, ${ }^{1}$ Jingjing Wu, ${ }^{1}$ Emily C O'Brien, ${ }^{1}$ Gregg C Fonarow, ${ }^{2}$ DaiWai M Olson, ${ }^{3}$ Lee H Schwamm, ${ }^{4}$ \\ Deepak L Bhatt, ${ }^{5}$ Eric E Smith, ${ }^{6}$ Robert E Suter, ${ }^{7}$ Deidre Hannah, Brianna Lindholm, \\ Lesley Maisch, Melissa A Greiner, ${ }^{1}$ Barbara L Lytle, ${ }^{1}$ Michael J Pencina, ${ }^{1}$ Eric D Peterson, ${ }^{1}$ \\ Adrian F Hernandez ${ }^{1}$
}

\section{ABSTRACT}

OBJECTIVE

To examine the association between warfarin treatment and longitudinal outcomes after ischemic stroke in patients with atrial fibrillation in community practice.

'Duke Clinical Research Institute, 2400 Pratt Street, Durham, NC, USA

2Division of Cardiology, University of California, Los Angeles, CA, USA ${ }^{3}$ Department of Neurology and Neurotherapeutics,

Neurological Surgery, University of Texas Southwestern Medical Center, Dallas, TX, USA

4Stroke Service, Massachusetts General Hospital and Harvard

Medical School, Boston, MA, USA '5righam and Women's Hospital Heart and Vascular Center and Harvard Medical School, Boston, MA, USA

${ }^{6}$ Hotchkiss Brain Institute, University of Calgary, Calgary, $\mathrm{AB}$, Canada

7The American Heart Association and University of Texas

Southwestern, Dallas, TX, USA

Correspondence to:

YXian ying.xian@duke.edu

Additional material is published online only. To view please visit the journal online (http://dx.doi. org/10.1136/bmj.h3786)

Cite this as: BMJ 2015;351:h3786 doi: 10.1136/bmj.h3786

Accepted: 21 June 2015
DESIGN

Observational study.

SETTING

Hospitals ( $n=1487$ ) participating in the Get With The

Guidelines (GWTG)-Stroke program in the United

States, from 2009 to 2011.

\section{PARTICIPANTS}

12552 warfarin naive atrial fibrillation patients admitted to hospital for ischemic stroke and treated with warfarin compared with no oral anticoagulant at discharge, linked to Medicare claims for longitudinal outcomes.

\section{MAIN OUTCOME MEASURES}

Major adverse cardiovascular events (MACE) and home time, a patient centered outcomes measure defined as the total number of days free from institutional care after discharge. A propensity score inverse probability weighting method was used to account for all differences in observed characteristics between treatment groups.

RESULTS

Among 12552 survivors of stroke, 11039 (88\%) were treated with warfarin at discharge. Warfarin treated patients were slightly younger and less likely to have a history of previous stroke or coronary artery disease but had similar severity of stroke as measured by the National

\section{WHAT IS ALREADY KNOWN ON THIS TOPIC}

Warfarin is recommended for the prevention of thromboembolism in patients with atrial fibrillation, on the basis of clinical trials in selected patient populations

However, the use and clinical benefit of warfarin for atrial fibrillation outside the clinical trial setting is not well defined, especially among older ischemic stroke patients

\section{WHAT THIS STUDY ADDS}

New prescription of warfarin in patients with atrial fibrillation after stroke was associated with a lower risk of major adverse cardiovascular events, all cause mortality, and readmission for ischemic stroke, as well as more institution-free home time

The clinical benefits associated with warfarin treatment were consistent across clinically relevant subgroups

These findings support the routine use of warfarin for eligible ischemic stroke patients with atrial fibrillation, including those over 80 years of age, women, those with more severe strokes, and those with comorbid conditions

Institutes of Health Stroke Scale. Relative to those not treated, patients treated with warfarin had more days at home (as opposed to institutional care) during the two years after discharge (adjusted home time difference 47.6 days, $99 \%$ confidence interval 26.9 to 68.2 ). Patients discharged on warfarin treatment also had a reduced risk of MACE (adjusted hazard ratio $0.87,99 \%$ confidence interval 0.78 to 0.98$)$, all cause mortality $(0.72,0.63$ to $0.84)$, and recurrent ischemic stroke $(0.63,0.48$ to 0.83$)$. These differences were consistent among clinically relevant subgroups by age, sex, stroke severity, and history of previous coronary artery disease and stroke.

\section{CONCLUSIONS}

Among ischemic stroke patients with atrial fibrillation, warfarin treatment was associated with improved long term clinical outcomes and more days at home.

CLINICAL TRIAL REGISTRATION

Clinical trials NCT02146274.

\section{Introduction}

Approximately 15 million people worldwide have a stroke each year. ${ }^{1}$ Atrial fibrillation is an important risk factor, accounting for approximately $15 \%$ of all strokes, and the highest incidence is in older patients. ${ }^{23}$ Clinical studies in selected trial populations have shown the efficacy of warfarin for prevention of stroke in patients

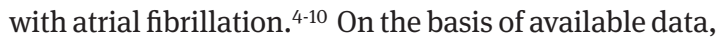
both European and US guidelines recommend adjusted dose warfarin treatment for stroke prevention in patients with non-valvular atrial fibrillation. ${ }^{11} 12$

Despite the existing evidence, the translation of clinical trial evidence into routine clinical practice is often challenging. Most clinical trials of warfarin for stroke prevention in patients with atrial fibrillation did not specifically enroll patients admitted to hospital for acute ischemic stroke. ${ }^{8-11}$ Moreover, clinical trials often have very strict protocols with regard to selection of patients, follow-up, monitoring, and dose adjustment to maximize a patient's time in the therapeutic range. Furthermore, most population based studies are from European countries, and the rate of anticoagulation after stroke in atrial fibrillation patients has improved dramatically in the past decade. ${ }^{1213}$ Whether the efficacy seen in clinical trials translates into effectiveness in contemporary practice is unclear, especially for secondary prevention among older patients who have already had an ischemic stroke and in clinically 
relevant subgroups that have higher risks of bleeding and more challenging warfarin management.

From a patient's perspective, an important measure of the benefit of anticoagulant treatment beyond survival is the prevention of recurrent events or prolonged hospital admissions. Focus groups identify "being alive, without recurrent stroke, and without being hospitalized for complications" as the most desirable outcome. ${ }^{14-16}$ This patient centered outcomes measure, which we have called "home time," has not been well studied as an endpoint in clinical trials. Using data from the American Heart Association/American Stroke Association Get With The Guidelines-Stroke (GWTG-Stroke) program linked with Centers for Medicare and Medicaid Services (CMS) claims data, we evaluated the association between warfarin treatment and longitudinal outcomes after ischemic stroke in the general atrial fibrillation population, as well as among clinically relevant subgroups of patients.

\section{Methods}

Study design and patient involvement

Details of the design and conduct of the Patient-Centered Research into Outcomes Stroke Patients Prefer and Effectiveness Research (PROSPER) study have been previously described. ${ }^{17}$ Briefly, PROSPER is a Patient-Centered Outcomes Research Institute (PCORI) sponsored project designed to help patients, physicians, and other stakeholders to make informed decisions about stroke care and to improve outcomes through innovative comparative effectiveness research. The study was conceived and designed by the PROSPER team, a multidisciplinary group of researchers at the Duke Clinical Research Institute, Massachusetts General Hospital, University of California at Los Angeles, University of Texas Southwestern Medical Center, Brigham and Women's Hospital, and University of Calgary, partnering with patient investigators and stakeholders representing the varied interests of patients, caregivers, physicians, nurses, healthcare systems, policy makers, governmental agencies, and professional societies.

To identify the most relevant research topics and meaningful outcomes, we worked with our patient co-investigators and conducted a series of focus groups with stroke survivors and administered a qualitative survey customized to stroke patients. Knowledge gained from these efforts was further reviewed by our patient co-investigators and stakeholder committee, who transformed these identified research needs into patient centered research questions to be studied, including the effectiveness of warfarin among ischemic stroke patients with atrial fibrillation.

The concept of patient involvement also translated to the study design and execution phases of the research. We provided basic educational materials and a research tutorial to help to encourage familiarity with research concepts and terminology. The pros and cons of different types of study design and research method were discussed with patient co-investigators, using laypersons' language to facilitate common understanding and solicit their feedback. We then revisited our aims and revised the research plans to align them better with things that patients cared about the most. As results emerged, we reviewed the results with patient co-investigators to obtain their perspectives and feedback to ensure that we presented the findings in the most effective way beyond the research community to general populations. Through these iterative processes, PROSPER patient co-investigators made high value contributions to both the design and the implementation of the study, while we retained the rigors of the scientific work.

\section{Data sources}

PROSPER builds on the American Stroke Association/ American Heart Association GWTG-Stroke program, which is an ongoing nationwide quality improvement initiative. ${ }^{1819}$ Standardized data collection in GWTGStroke includes patients' demographics, medical history, diagnostic testing, brain imaging, in-hospital treatment, discharge drugs, and inpatient outcomes. The eligibility of each admission is confirmed through chart review. The validity and reliability of data collection in the GWTGStroke database has been reported in previous research. ${ }^{20}$

As GWTG-Stroke is an inpatient registry, we linked GWTG-Stroke data to Medicare claims by matching on a series of indirect identifiers, including admission date, discharge date, patient's age or date of birth, and sex, for the assessment of longitudinal outcomes. This linkage method, developed by our analytic team, has been successfully completed and validated using Medicare inpatient claims..$^{21}$ Previous work has shown that patients in the linked GWTG-Stroke/CMS database are representative of the national Medicare ischemic stroke population. ${ }^{22}$ Quintiles Inc serves as the data collection (through its patient management tool) and coordination center for GWTG-Stroke. The Duke Clinical Research Institute serves as the data analysis center and has an agreement to analyze the aggregate de-identified data for research purposes.

\section{Study population}

Our analysis included Medicare fee for service beneficiaries admitted to GWTG-Stroke hospitals for acute ischemic stroke and discharged alive with documented persistent or paroxysmal atrial fibrillation or flutter between 1 January 2009 and 31 December 2011. Each patient had at least one year of follow-up after the index hospital discharge, using hospital claims data submitted to CMS through December 2012. Figure 1 shows details of the study's inclusion and exclusion criteria. Briefly, we excluded patients with documented contraindications for anticoagulation treatment, as well as those who received comfort measures only or were transferred out to other hospitals. To avoid prevalent user bias, we further excluded patients receiving chronic anticoagulation treatment before the index hospital admission. ${ }^{23}$ We abstracted the discharge drug treatment from the medical record of the index hospital admission. We divided patients into two groups according to discharge drug treatment: patients treated with warfarin versus those not treated with any oral anticoagulant at discharge. Patients who refused warfarin but had no contraindications were considered eligible for warfarin but did not receive the treatment $(n=1162)$. 


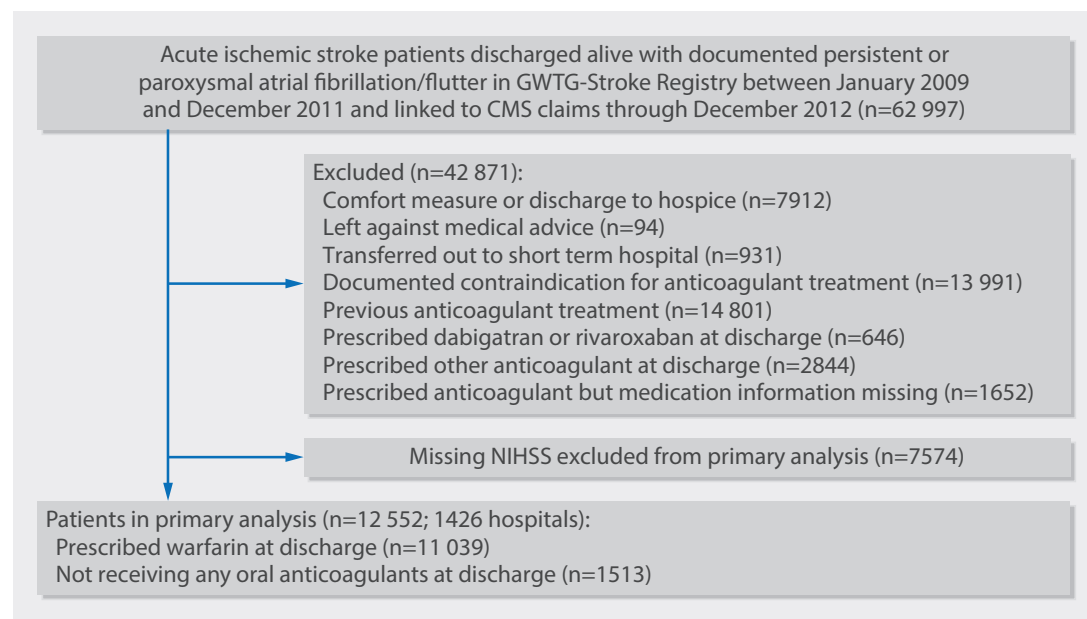

Fig 1 | Study population from initial cohort, through exclusions, to final population. CMS=Centers for Medicare and Medicaid Services; GWTG-Stroke=Get With The GuidelinesStroke; NIHSS=National Institutes of Health Stroke Scale

Novel oral anticoagulants such as dabigatran and rivaroxaban were not recorded in GWTG-Stroke until October 2011. Therefore, we also excluded patients discharged on novel oral anticoagulants or other agents such as low molecular weight heparin or fondaparinux. As the National Institutes of Health Stroke Scale (NIHSS) score (a measure of neurologic deficits ranging from 0 to 42, with a higher score indicating greater severity of stroke) is a critical predictor of outcomes in acute ischemic stroke, ${ }^{2425}$ our primary study population consisted of 12552 patients with complete NIHSS data from 1487 GWTG-Stroke hospitals in the United States.

\section{Outcome measures}

We conducted focus group and surveys of patients to identify outcomes most relevant and meaningful to patients who have survived stroke. ${ }^{17}$ The most frequent and important outcome identified was "alive at home, without recurrent stroke, and without being hospitalized for complications."15 16 On the basis of the interpretation of patients' responses and further refinement by patient co-investigators, we translated this concept into home time, which we defined as the total number of days alive and out of the hospital or a skilled nursing facility within two years after the index hospital discharge. We calculated home time by using Medicare inpatient care, inpatient rehabilitation facility, and skilled nursing home claims. ${ }^{26}$ Similarly, a major adverse cardiovascular event (MACE) is a composite measure of all cause mortality or cardiovascular readmission, reflecting patients' desire of "being alive at home, without recurrent stroke, or being hospitalized for complications." Secondary outcomes included all cause mortality, cardiovascular readmission, ischemic stroke readmission, and hemorrhagic stroke readmission.

Where applicable, we determined the date of death through the Medicare denominator files. We ascertained the date and cause of readmission from the hospital claims data submitted to CMS, with the international classification of diseases, ninth revision, clinical modification (ICD-9-CM) primary diagnosis code of 433.x1 or 434.x1 for ischemic stroke and 430 or 431 for hemorrhagic stroke and the Agency for Healthcare Research and Quality's clinical classification software diagnosis category 96-118 for cardiovascular admission. The clinical classification software is a diagnosis and procedure categorization scheme developed by the Agency for Healthcare Research and Quality to identify disease specific or procedure specific hospital admission by grouping individual ICD-9-CM codes into clinically meaningful categories. ${ }^{27}$ The use of ICD-9-CM codes 433.x1, 434.x1, 430, and 431 has been proposed by the American Heart Association/American Stroke Association for defining stroke using ICD-9-CM codes and for CMS stroke core measures..$^{28-30}$

\section{Statistical analyses}

Our pre-specified statistical analysis plan has been previously described. ${ }^{17}$ To summarize, we used means, medians, and percentages to describe the distribution of continuous and categorical variables between patients discharged on warfarin and those discharged not on any oral anticoagulants. We defined warfarin treatment as warfarin prescribed at the time of hospital discharge, as documented in GWTG-Stroke. We used standardized difference to compare baseline characteristics between patient cohorts. An absolute standardized difference greater than $10 \%$ indicates significant imbalance of a covariate, whereas a smaller value supports the assumption of balance between treatment groups. ${ }^{31}$

Unlike randomized clinical trials, the decision to treat in "real world" practice is often based on prognostic factors. Therefore, the effectiveness estimate for warfarin might be confounded as a result of treatment selection. We used a stabilized inverse probability weighting approach to control for potential bias. Inverse probability weighting is an extension of the propensity score method used to summarize the conditional probability of assignment for a treatment. ${ }^{3233}$ Using this method, the weights are the inverse probability of assigning a treatment derived from a logistic model with warfarin treatment as the dependent variable and all observed patient level and hospital level characteristics as the independent variables. These variables included age; sex; race/ethnicity; history of previous stroke/transient ischemic attack, coronary artery disease/myocardial infarction, carotid stenosis, peripheral vascular disease, hypertension, dyslipidemia, diabetes, or smoking; emergency medical services transportation; NIHSS at presentation; hospital bed size; annual ischemic stroke volume; American Heart Association/American Stroke Association/Joint Commission certification as a primary stroke center; academic status; rural/urban location; and calendar year. Each patient was then weighted by the inverse of the estimated probability of the treatment received.

We then used inverse probability weighting regression models weighted with warfarin treatment as the independent variable to estimate the adjusted associations between warfarin treatment and each outcome estimated, controlling for other discharge drugs as covariates. Discharge drugs included antihypertensives, 
statins, and antiplatelets (aspirin, aspirin/dipyridamole, clopidogrel, aspirin/clopidogrel dual antiplatelet therapy, and other antiplatelet agents). Owing to the presence of over-dispersion in home time and the fact that not all patients had the same length of time in follow-up (for example, patients lost Medicare fee for service coverage or transitioned to Medicaid), we used a negative binomial regression with an offset for log of the proportion of follow-up time to model home time. We used separate Cox proportional hazards models for time to event data including MACE, all cause mortality, all cause readmission, cardiovascular readmission, ischemic stroke readmission, and hemorrhagic stroke readmission.

In addition to the general population of ischemic stroke patients with atrial fibrillation, we did these analyses in clinically relevant subgroups by age (65-80 and $>80$ years), sex, history of previous stroke/transient ischemic attack and coronary artery disease/myocardial infarction, and stroke severity as measured by NIHSS (mild 0-5, moderate 6-14, and severe 15-42). Our analyses also accounted for within hospital clustering by using random effects for negative binomial models and robust sandwich estimators for Cox proportional hazards models. In a sensitivity analysis, we included $7574(37.6 \%)$ patients with missing NIHSS data and extended our study population to the entire study cohort (total $n=20126$ ). We used a multiple imputation method to impute missing NIHSS values. The characteristics of the patient cohorts including those without NIHSS recorded and only patients with NIHSS recorded are shown in supplementary table A.

All analyses followed the intention to treat principle. All $\mathrm{P}$ values are two sided. Owing to multiple outcomes and subgroups assessed, we report 99\% confidence intervals and considered $P$ values less than 0.01 to be statistically significant. We used SAS version 9.3 for all statistical analyses.

\section{Results}

Of 12552 patients with ischemic stroke discharged alive with persistent or paroxysmal atrial fibrillation or flutter and untreated with oral anticoagulation before admission, 11039 (87.9\%) were treated with warfarin at discharge. Table 1 shows the baseline demographic, clinical, and hospital characteristics according to warfarin prescription at discharge. The age distribution of the study cohort is shown in supplementary figure A. Compared with patients not receiving warfarin at discharge, those treated with warfarin were slightly younger and were less likely to have a history of previous stroke or coronary artery disease. Fewer patients receiving warfarin were treated with aspirin/dipyridamole, clopidogrel, or aspirin/clopidogrel dual antiplatelet therapy at discharge. By contrast, both groups had similar stroke severity as measured by NIHSS (median 6 (interquartile range 2-13) v 5 (2-12)). Results from the warfarin treatment propensity model indicated that older patients with a history of previous stroke, coronary artery disease, diabetes, or smoking were less likely to be treated with warfarin at discharge, whereas patients treated at primary stroke centers were more likely to receive warfa- rin (supplementary table B). After inverse probability weighting adjustment, patients were well matched on all observed characteristics, with an absolute standardized difference less than 10\% (supplementary figure B).

\section{MACE and home time}

The incidence of MACE was $54.7 \%$ in patients treated with warfarin at discharge, compared with $66.8 \%$ in those not on warfarin (unadjusted $\mathrm{P}<0.001$ ). On average, patients receiving warfarin at discharge had 86 more days of home time in the two year follow-up period than those not receiving warfarin at discharge (unadjusted $\mathrm{P}<0.001$ ) (table 2). After weighting by the inverse probability of treatment and control for other discharge drugs, patients treated with warfarin at discharge had a significantly lower risk of MACE over two years (adjusted hazard ratio 0.87, 99\% confidence interval 0.78 to 0.98 ) and were more likely to spend more days alive and out of the hospital or a skilled nursing facility (adjusted home time difference 47.6, 99\% confidence interval 26.9 to 68.2 , days) than those not receiving any oral anticoagulants at discharge (table 2, fig 2). The association of warfarin with primary outcomes of MACE (adjusted hazard ratio $0.87,0.80$ to 0.96 ) and home time (48.5, 36.5 to 60.4 , days) remained essentially unchanged after inclusion of patients with missing NIHSS data.

We also evaluated clinical outcomes for clinically relevant subgroups. Of 12552 patients with ischemic stroke, 6590 (52.5\%) were older than 80 years, $7538(60.1 \%)$ were women, $6068(48.3 \%)$ had an NIHSS of 0-5, 3931 (31.3\%) had as NIHSS of 6-14, 2553 (20.3\%) had an NIHSS of $15-42,3963(31.6 \%)$ had a history of coronary artery disease, and 2990 (21.6\%) had had a previous stroke/ transient ischemic attack. We found a similar trend of lower risk of MACE and more home time associated with warfarin treatment in each clinically relevant subgroup, although the benefit in terms of MACE seemed to be more prominent in patients who were older, were female, presented with more severe stroke, and had no history of coronary artery disease (fig 3).

\section{Secondary outcomes}

The unadjusted incidences of all cause mortality, all cause readmission, ischemic stroke readmission, and cardiovascular readmission were lower among patients treated with warfarin at discharge (table 2). In contrast, patients receiving warfarin had a higher unadjusted incidence of hemorrhagic stroke than did those not receiving anticoagulation treatment during follow-up (1.4\% $v 1.1 \%$, $\mathrm{P}=0.50$ ). After inverse probability weighting adjustment and control for other discharge drugs, discharge warfarin treatment was associated with lower risk of all cause mortality (adjusted hazard ratio $0.72,0.63$ to 0.84 ) and ischemic stroke readmission $(0.63,0.48$ to 0.83$)$ but no statistically significant difference in hemorrhagic stroke $(1.37,0.61$ to 3.06$)$ in the two years after discharge.

\section{Discussion}

Using a large nationwide contemporary registry of patients admitted to hospital with acute ischemic stroke, we found that new prescription of warfarin treatment in patients with atrial fibrillation was associated with a lower risk of 


\begin{tabular}{|c|c|c|c|}
\hline Variable & Warfarin $(n=11039)$ & $\begin{array}{l}\text { No oral anticoagulant } \\
(n=1513)\end{array}$ & $\begin{array}{l}\text { Standardized } \\
\text { difference (\%) }\end{array}$ \\
\hline \multicolumn{4}{|l|}{ Patients } \\
\hline Mean (SD) age, years & $80.1(7.5)$ & $83.1(7.9)$ & 39.1 \\
\hline Women & $6582(59.6)$ & $956(63.2)$ & 7.3 \\
\hline \multicolumn{4}{|l|}{ Race/ethnicity: } \\
\hline White & $9412(85.3)$ & $1291(85.3)$ & \multirow{5}{*}{4.5} \\
\hline Black & $699(6.3)$ & $105(6.9)$ & \\
\hline Asian & $221(2.0)$ & $26(1.7)$ & \\
\hline Hispanic & $379(3.4)$ & $54(3.6)$ & \\
\hline Other & $328(3.0)$ & $37(2.4)$ & \\
\hline \multicolumn{4}{|l|}{ History of: } \\
\hline Previous stroke & $1630(14.8)$ & $312(20.6)$ & 15.4 \\
\hline Previous transient ischemic attack & $901(8.2)$ & $147(9.7)$ & 5.4 \\
\hline Carotid stenosis & $443(4.0)$ & $70(4.6)$ & 3.0 \\
\hline Coronary artery disease/myocardial infarction & $3402(30.8)$ & $561(37.1)$ & 13.3 \\
\hline Hypertension & $8838(80.1)$ & $1212(80.1)$ & 0.1 \\
\hline Dyslipidemia & $4879(44.2)$ & $582(38.5)$ & 11.7 \\
\hline Peripheral vascular disease & $541(4.9)$ & $98(6.5)$ & 6.8 \\
\hline Diabetes mellitus & $2768(25.1)$ & $426(28.2)$ & 7.0 \\
\hline Smoking & $785(7.1)$ & $105(6.9)$ & 0.7 \\
\hline Mode of arrival, emergency medical services from scene & $6283(56.9)$ & $916(60.5)$ & 7.4 \\
\hline \multicolumn{4}{|l|}{ National Institutes of Health Stroke Scale: } \\
\hline Median (interquartile range) & $6(2-13)$ & $5(2-12)$ & \multirow{4}{*}{1.8} \\
\hline $0-5$ & $5299(48.0)$ & $769(50.8)$ & \\
\hline 6-14 & $3483(31.6)$ & $448(29.6)$ & \\
\hline $15-42$ & $2257(20.4)$ & $296(19.6)$ & \\
\hline \multicolumn{4}{|l|}{ Discharge drugs: } \\
\hline Oral anticoagulant & $11039(100)$ & $0(0)$ & - \\
\hline Antiplatelet & $5912(53.6)$ & $1356(89.6)$ & 87.3 \\
\hline Aspirin only & $5086(46.1)$ & $714(47.2)$ & 2.2 \\
\hline Aspirin/dipyridamole & $111(1.0)$ & $112(7.4)$ & 32.3 \\
\hline Clopidogrel only & $398(3.6)$ & $187(12.4)$ & 32.7 \\
\hline Aspirin and clopidogrel & $298(2.7)$ & $332(21.9)$ & 61.2 \\
\hline Other antiplatelet & $19(0.2)$ & $11(0.7)$ & 8.3 \\
\hline Antihypertensive & $8919(80.8)$ & $1219(80.6)$ & 0.6 \\
\hline Statin for low density lipoprotein cholesterol $\geq 100 \mathrm{mg} / \mathrm{dL}$ & 4710/5082 (92.7) & $604 / 694(87.0)$ & 18.8 \\
\hline \multicolumn{4}{|l|}{ Hospitals } \\
\hline Mean (SD) No of beds, & $441(292)$ & $420(306)$ & 6.8 \\
\hline Mean (SD) annual stroke volume & $255(148)$ & $235(149)$ & 13.6 \\
\hline Hospital type, academic & $6440(58.3)$ & $856(56.6)$ & 3.6 \\
\hline Primary stroke center & $6427(58.2)$ & $779(51.5)$ & 13.6 \\
\hline Rural hospital & $486(4.4)$ & $91(6.0)$ & 7.3 \\
\hline
\end{tabular}

\begin{tabular}{|c|c|c|c|c|c|}
\hline Outcome* & Warfarin $(n=11039)$ & $\begin{array}{l}\text { No oral anticoagulant } \\
(\mathrm{n}=1513)\end{array}$ & $\begin{array}{l}\text { Unadjusted hazard } \\
\text { ratio }(99 \% \mathrm{Cl})\end{array}$ & $\begin{array}{l}\text { Adjusted hazard } \\
\text { ratio }(99 \% \mathrm{Cl})\end{array}$ & $P$ value \\
\hline No (\%) MACE & $5683(54.7)$ & $952(66.8)$ & 0.73 (0.67 to 0.80$)$ & 0.87 (0.78 to 0.98$)$ & 0.003 \\
\hline Mean (SD) home time, days & $468.3(254.7)$ & $389.0(266.7)$ & 86.1 (68.0 to 104.1)† & $47.6(26.9$ to 68.2$) \dagger$ & $<0.001$ \\
\hline No (\%) all cause mortality & $3285(32.4)$ & $699(50.0)$ & $0.57(0.51$ to 0.63$)$ & $0.72(0.63$ to 0.84$)$ & $<0.001$ \\
\hline \multicolumn{6}{|l|}{ Readmission: } \\
\hline All cause & $6794(64.5)$ & 951 (65.9) & $0.88(0.81$ to 0.97$)$ & 1.01 (0.91 to 1.13$)$ & 0.79 \\
\hline Ischemic stroke & $814(7.9)$ & $169(11.8)$ & 0.58 (0.47 to 0.72$)$ & 0.63 (0.48 to 0.83$)$ & $<0.001$ \\
\hline Hemorrhagic stroke & $140(1.4)$ & $16(1.1)$ & $1.10(0.56$ to 2.16$)$ & $1.37(0.61$ to 3.06$)$ & 0.31 \\
\hline Cardiovascular & $3673(35.3)$ & $515(36.0)$ & 0.87 (0.77 to 0.98) & 1.00 (0.86 to 1.16$)$ & 0.97 \\
\hline
\end{tabular}

MACE, all cause mortality, and readmission for ischemic stroke, as well as more institution-free home time. The clinical benefits associated with warfarin treatment were consistent across clinically relevant subgroups and risk adjustment methods. Collectively, our findings suggest that the efficacy of anticoagulation in clinical trials may translate into real world clinical practice and support the current guideline recommendations of warfarin treatment as an anticoagulation approach for the prevention of recurrent stroke in patients with atrial fibrillation. ${ }^{112}$ 


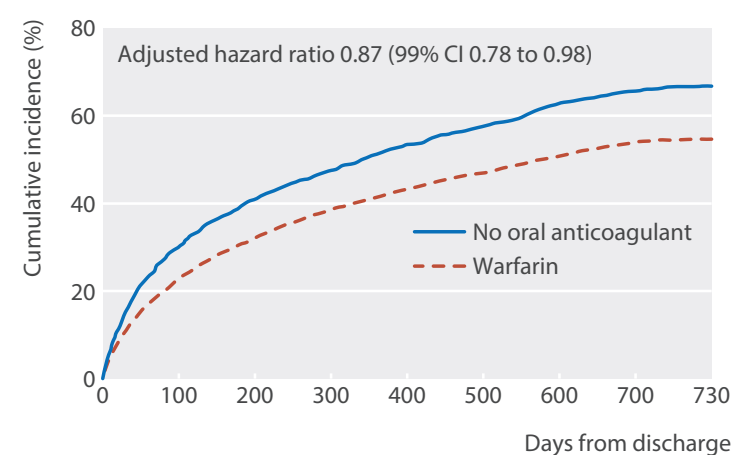

Fig 2 | Cumulative incidences for major adverse cardiovascular event during two year follow-up for patients receiving warfarin versus no oral anticoagulant

Warfarin has been shown to be superior to placebo in primary stroke prevention trials among patients with atrial fibrillation, but the data on secondary prevention among patients who have already had an ischemic stroke and in clinically relevant subgroups, including patients older than 80 years and women, are much more limited..$^{7-11}$ A meta-analysis of six randomized clinical trials found a $64 \%$ reduction in stroke associated with
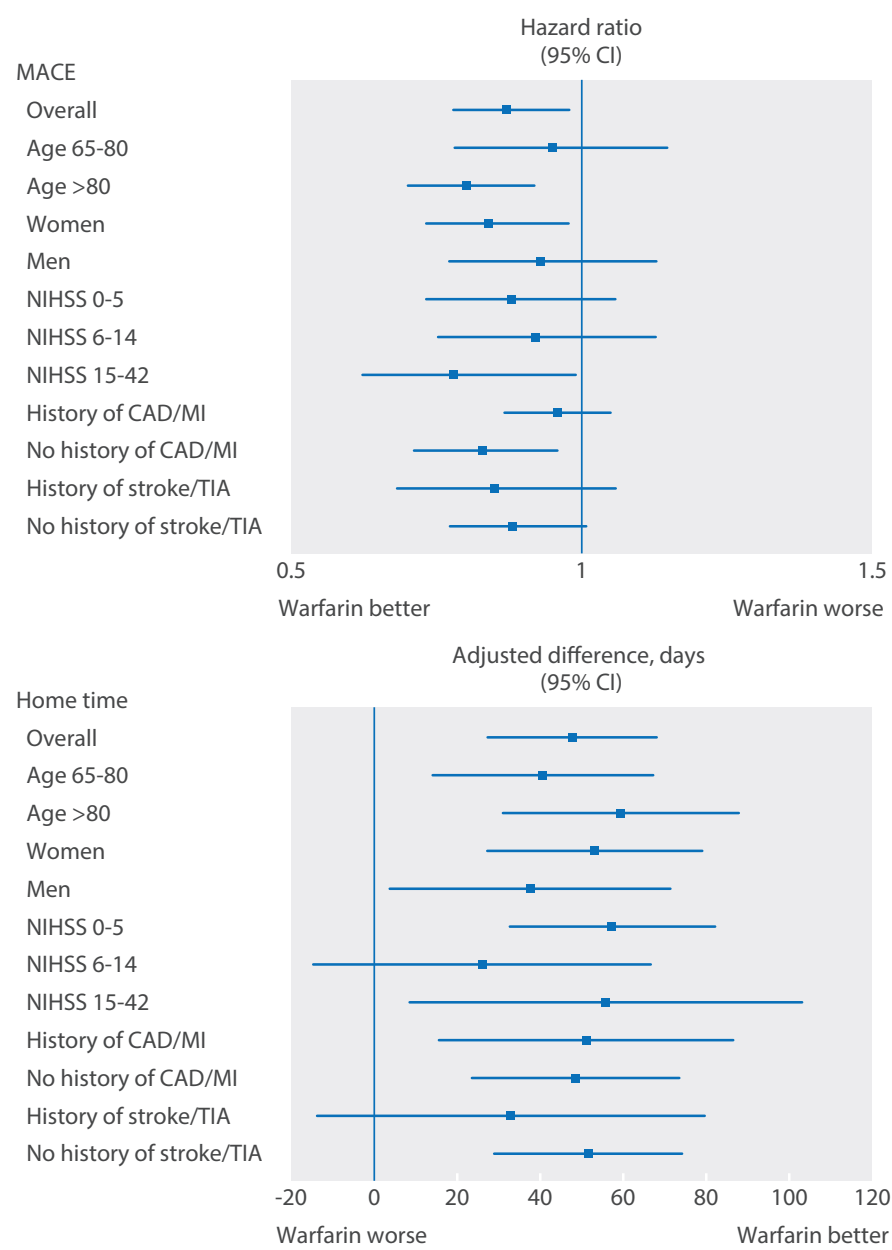

Fig 3 | Major adverse cardiovascular event (MACE) and home time according to warfarin treatment at discharge, overall and in clinically relevant subgroups. $C A D=$ coronary artery disease; $\mathrm{MI}=$ myocardial infarction; NIHSS=National Institutes of Health Stroke Scale; TIA=transient ischemic attack adjusted dose warfarin treatment in selected atrial fibrillation populations. ${ }^{34}$ We evaluated the clinical effectiveness of warfarin treatment for secondary prevention among older ischemic stroke patients with atrial fibrillation in community practice. We found that those treated with warfarin at discharge had 86 more days (48 days after adjustment) on average free from institutional care than did their untreated counterparts during the two years after their stroke. We also observed a favorable association of warfarin treatment with reduced risks of MACE, all cause mortality, and recurrent ischemic stroke. Although the observed benefits are smaller than those reported in clinical trials, our observations are not surprising, given that the efficacy of warfarin treatment is dependent on the the intensity of anticoagulation. ${ }^{35}$ This partially mitigates the concern that the benefits of warfarin reported in clinical trials may not translate into usual clinical care, particularly among older patients and those with multiple comorbid conditions.

Importantly, we found that the benefits associated with warfarin treatment were consistent across clinically relevant groups by age, sex, stroke severity, and history of stroke and coronary artery disease. A salient finding is that older patients (age >80), women, and those with more severe stroke (NIHSS 15-42) seemed to have greater benefit from warfarin treatment, yet these patients were less likely to be treated with warfarin in clinical practice. Reasons for the treatment-risk paradox are complex, but they might be related to physicians' misperceptions, uncertainty of risk and benefit, and reluctance to cause harm in patients at high risk who are often underrepresented in clinical trials. ${ }^{3637}$ Our study attempted to fill this gap by evaluating the clinical effectiveness of warfarin treatment in clinically relevant subgroups. Although we did not have information on the exact clinical circumstance of a treatment decision, physicians may tend to be risk averse and not prescribe more aggressive treatment to high risk patients who might be perceived to have a poor prognosis. An objective and precise benefit/risk prediction tool will help to overcome the paradox by promoting a better understanding of the clinical factors contributing to patients' outcomes.

To the best of our knowledge, our research is the first comparative effectiveness study on warfarin use after stroke with atrial fibrillation that has incorporated input from patients throughout the entire research process, reflecting their healthcare priorities, choice of outcome measures, and input into the statistical analysis plan. One of the strengths of our study is the integration of home time, which is a patient centered measure of longitudinal functional outcomes prioritized by stroke survivors and stakeholders. ${ }^{1516}$ Home time is a simple objective measure and is closely associated with the modified Rankin scale and Barthel index. ${ }^{2638}$ Owing to data limitations, we could not fully distinguish whether home time meant that patients were living in their own home, living at a relative's or friend's house, at home with home care services, at home with other community support, in assisted living, homeless, or exceeding the Medicare benefit for post-acute care and transitioning to Medicaid because of poverty. We also recognize 
that being at home does not equate to the absence of disability. Even with these imperfections, home time indicates the absence of institutional care, which is a highly valued health state prioritized by stroke survivors. ${ }^{15} 16$ More importantly, home time reflects the overall health status of the patient following stroke, is meaningful from patients' perspectives, and is easy to measure using claims data.

The benefit of more days at home with warfarin treatment could be partially explained by the lower MACE rates-in particular, reduction in death and recurrent ischemic stroke events after discharge. Despite numerically more bleeding events in patients treated with warfarin, the association between warfarin treatment and hemorrhagic stroke was not statistically significant after risk adjustment. This finding should be interpreted with caution, as our study was not adequately powered for assessing hemorrhagic stroke, owing to the overall low incidence. Nevertheless, the fact that the overall incidence of hemorrhagic stroke was low is reassuring, suggesting that the net benefit of warfarin is likely to be greater than the effect of bleeding, even if the difference was statistically significant.

\section{Limitations of study}

Our study should be interpreted in the context of certain limitations. Firstly, this was an observational analysis of registry data. Despite use of propensity score techniques to minimize observed confounding, residual and unmeasured confounding may exist. To help to limit bias, we used a new user design as well as a pre-specified study protocol and statistical analysis plan similar to those used in clinical trials. Secondly, our analysis was based on warfarin status at discharge and we do not have data on adherence to treatment and international normalized ratio during follow-up. Early discontinuation of treatment or poor adherence could potentially bias some of these findings. This being said, the level of anticoagulation with warfarin treatment is often managed less well in community practice than in clinical trials. A recent study indicates that $50 \%$ of Medicare beneficiaries who were advised to take an oral anticoagulant filled only one prescription. ${ }^{39}$ Therefore, it could be argued that our study represents an intention to treat comparison that reflects real world effectiveness. The benefit associated with warfarin treatment would have been even larger if higher adherence could be achieved. Thirdly, our study is restricted to fee for service Medicare beneficiaries and, as a result, may not be generalizable to other populations. Fourthly, we were unable to report direct functional outcome measures such as disability and the modified Rankin scale. Instead, our study was able to report home time, an objective outcome correlated closely with the modified Rankin scale and increasingly being examined in stroke research. ${ }^{26}$ Furthermore, home time better reflects patients' preference of living independently in the community. Presenting the benefit of warfarin in patient centric terms rather than through traditional clinical endpoints and outcomes may help to improve adherence to anticoagulant treatment. Finally, GWTG-Stroke is a voluntary program. Despite being the largest stroke registry in the United States, capturing more than a third of annual ischemic stroke admissions, these results might not be extrapolated to patients treated in non-GWTG-Stroke hospitals or to patients in other countries.

\section{Conclusions}

Among ischemic stroke patients with atrial fibrillation, new prescription of warfarin treatment at discharge was associated with lower mortality, fewer major adverse cardiovascular events, fewer recurrent ischemic stroke events, and more time spent at home during the two years after stroke. These findings support the routine use of warfarin treatment for eligible ischemic stroke patients with atrial fibrillation, including those over 80 years of age, women, those with more severe strokes, and those with comorbid conditions. With the availability of new oral anticoagulants in clinical practice, further studies should evaluate the safety and clinical effectiveness of other anticoagulation regimens.

We thank Erin Hanley, Duke Clinical Research Institute, for editorial assistance. She did not receive compensation for her assistance apart from her employment at the institution where this study was conducted.

Contributors: All authors were involved in the study design, data analysis, and revision of the manuscript. All authors read and approved the final manuscript. YX is the guarantor.

Funding: This work was supported by an award (CE-1304) from the Patient-Centered Outcomes Research Institute (PCORI). The funders of the study had no role in the study design, data collection, data analysis, data interpretation, writing of the report, or the decision to submit the article for publication.

Competing interests: All authors have completed the ICMJE uniform disclosure form at www.icmje.org/coi disclosure.pdf (available on request from the corresponding author) and declare: support by PCORI for the submitted work. YX reports research funding to Duke Clinical Research Institute from the American Heart Association, Daiichi Sankyo, Janssen Pharmaceuticals, and Genentech. GCF serves as a member of the Get With The Guidelines (GWTG) Steering Committee; receives research support from the National Institutes of Health and PCORI; and is an employee of the University of California, which holds a patent on retriever devices for stroke. LHS is the principal investigator of an investigator initiated study of extended window intravenous thrombolysis funded by the National Institutes of Neurological Disorders and Stroke (clinicaltrials.gov/show/ NCT01282242), for which Genentech provides alteplase free of charge to Massachusetts General Hospital as well as supplemental per patient payments to participating sites; he also serves as chair of the American Heart Association/American Stroke Association GWTG Stroke Clinical Work Group, as a stroke systems consultant to the Massachusetts Department of Public Health, and as a scientific consultant regarding trial design and conduct to Lundbeck (international steering committee, DIAS3, 4 trial) and Penumbra (data and safety monitoring committee, Separator 3D trial). DLB serves on advisory boards for Cardax, Elsevier Practice Update Cardiology, Medscape Cardiology, and Regado Biosciences; on the Board of Directors of Boston VA Research Institute and Society of Cardiovascular Patient Care; is chair of the American Heart Association GWTG Steering Committee; serves on data monitoring committees for Duke Clinical Research Institute, Harvard Clinical Research Institute, Mayo Clinic, and Population Health Research Institute; has received honorariums from the American College of Cardiology (senior associate editor, clinical trials and news, acc.org), Belvoir Publications (editor in chief, Harvard Heart Letter), Duke Clinical Research Institute (clinical trial steering committees), Harvard Clinical Research Institute (clinical trial steering committee), HMP Communications (editor in chief, Journal of Invasive Cardiology), Journal of the American College of Cardiology (associate editor; section editor, pharmacology), Population Health Research Institute (clinical trial steering committee), Slack Publications (chief medical editor, cardiology today's intervention), WebMD (CME steering committees); is deputy editor of Clinical Cardiology; has received 
research funding from Amarin, AstraZeneca, Bristol-Myers Squibb, Eisai, Ethicon, Forest Laboratories, Ischemix, Medtronic, Pfizer, Roche, Sanofi Aventis, and The Medicines Company; and has conducted unfunded research for FlowCo, PLx Pharma, and Takeda. EES serves as a member of the GWTG Steering Committee. RES is employed as the vice president of quality and HIT by the American Heart Association/American Stroke Association, which receives grant funding from multiple pharmaceutical and other sources. EDP has received research grants from Lilly, Johnson \& Johnson, Bristol-Myers Squibb, Sanofi-Aventis, and Merck-Schering Plough partnership; and serves as principal investigator of the data analytic center for the American Heart Association/American Stroke Association's GWTG program. AFH has received research grants from Amgen, Bristol Myers Squibb, GlaxoSmithKline, Janssen, Novartis, and Portola Pharmaceuticals and honorariums from Amgen, GlaxoSmithKline, lanssen, and Novartis.

Ethical approval: The institutional review board of the Duke University Health System approved this study.

Data sharing: No additional data available.

Transparency declaration: The lead author (the manuscript's guarantor) affirms that the manuscript is an honest, accurate, and transparent account of the study being reported; that no important aspects of the study have been omitted; and that any discrepancies from the study as planned (and, if relevant, registered) have been explained.

This is an Open Access article distributed in accordance with the Creative Commons Attribution Non Commercial (CC BY-NC 4.0) license, which permits others to distribute, remix, adapt, build upon this work non-commercially, and license their derivative works on different terms, provided the original work is properly cited and the use is noncommercial. See: http://creativecommons.org/licenses/by-nc/4.0/.

1 World Health Organization. Global burden of stroke. In: The atlas of heart disease and stroke. WHO, 2004 (available at www.who.int/ cardiovascular_diseases/en/cvd_atlas_15_burden_stroke.pdf?ua=1).

2 Goldstein LB, Bushnell CD, Adams RJ, et al. Guidelines for the primary prevention of stroke: a guideline for healthcare professionals from the American Heart Association/American Stroke Association. Stroke 2011; $42: 517-84$

3 Wolf PA, Abbott RD, Kannel WB. Atrial fibrillation as an independent risk factor for stroke: the Framingham Study. Stroke 1991;22:983-8.

4 Hylek EM, Go AS, Chang Y, et al. Effect of intensity of oral anticoagulation on stroke severity and mortality in atrial fibrillation. $N$ Engl I Med 2003:349:1019-26.

5 EAFT (European Atrial Fibrillation Trial) Study Group. Secondary prevention in non-rheumatic atrial fibrillation after transient ischaemic attack or minor stroke. Lancet 1993:342:1255-62.

6 Adjusted-dose warfarin versus low-intensity, fixed-dose warfarin plus aspirin for high-risk patients with atrial fibrillation: Stroke Prevention in Atrial Fibrillation III randomised clinical trial. Lancet 1996;348:633-8.

7 Petersen P, Boysen G, Godtfredsen J, et al. Placebo-controlled, randomised trial of warfarin and aspirin for prevention of thromboembolic complications in chronic atrial fibrillation: the Copenhagen AFASAK study Lancet 1989:1:175-9.

8 Ezekowitz MD, Bridgers SL, James KE, et al. Warfarin in the prevention of stroke associated with nonrheumatic atrial fibrillation. N Engl J Med 1992;327:1406-12

9 The Boston Area Anticoagulation Trial for Atrial Fibrillation Investigators. The effect of low-dose warfarin on the risk of stroke in patients with nonrheumatic atrial fibrillation. N Engl J Med 1990;323:1505-11.

10 Connolly SJ, Laupacis A, Gent M, et al. Canadian Atrial Fibrillation Anticoagulation (CAFA) Study. J Am Coll Cardiol 1991;18:349-55.

11 Camm AJ, Kirchhof P, Lip GY, et al. Guidelines for the management of atrial fibrillation: the Task Force for the Management of Atrial Fibrillation of the European Society of Cardiology (ESC). Eur Heart) 2010;31:2369-429.

12 Andersen KK, Olsen TS. Reduced poststroke mortality in patients with stroke and atrial fibrillation treated with anticoagulants: results from a Danish quality-control registry of 22179 patients with ischemic stroke. Stroke 2007;38:259-63.

13 Weimar C, Benemann J, Katsarava Z, et al. Adherence and quality of oral anticoagulation in cerebrovascular disease patients with atrial fibrillation. Eur Neurol 2008;60:142-8.

14 Kernan WN, Ovbiagele B, Black HR, et al. Guidelines for the prevention of stroke in patients with stroke and transient ischemic attack: a guideline for healthcare professionals from the American Heart Association/American Stroke Association. Stroke 2014:45:2160-236.
15 O’Brien EC, Xian Y, Fonarow GC, et al. Clinical commentary on "Certain uncertainty: life after stroke from the patient's perspective". Circ Cardiovasc Qual Outcomes 2014;7:970.

16 Hannah D, Lindholm B, Maisch L. Certain uncertainty: life after stroke from the patient's perspective. Circ Cardiovasc Qual Outcomes 2014;7:968-9.

17 Xian Y, O’Brien EC, Fonarow GC, et al. Patient-Centered Research into Outcomes Stroke Patients Prefer and Effectiveness Research (PROSPER): implementing the patient-driven research paradigm to aid decision making in stroke care. Am Heart J 2015;170:36-45.e11.

18 Fonarow GC, Reeves MJ, Smith EE, et al. Characteristics, performance measures, and in-hospital outcomes of the first one million stroke and transient ischemic attack admissions in Get With The GuidelinesStroke. Circ Cardiovasc Qual Outcomes 2010;3:291-302.

19 Schwamm LH, Fonarow GC, Reeves Ml, et al. Get With the Guidelines-Stroke is associated with sustained improvement in care for patients hospitalized with acute stroke or transient ischemic attack. Circulation 2009:119:107-15.

20 Xian Y, Fonarow GC, Reeves MJ, et al. Data quality in the American Heart Association Get With The Guidelines-Stroke (GWTG-Stroke): results from a national data validation audit. Am Heart) 2012:163:392-8.e1.

21 Hammill BG, Hernandez AF, Peterson ED, et al. Linking inpatient clinical registry data to Medicare claims data using indirect identifiers. Am Heart / 2009;157:995-1000.

22 Reeves MJ, Fonarow GC, Smith EE, et al. Representativeness of the Get With The Guidelines-Stroke Registry: comparison of patient and hospital characteristics among Medicare beneficiaries hospitalized with ischemic stroke. Stroke 2012;43:44-9.

23 Ray WA. Evaluating medication effects outside of clinical trials: new-user designs. Am / Epidemiol 2003:158:915-20.

24 Fonarow GC, Pan W, Saver JL, et al. Comparison of 30-day mortality models for profiling hospital performance in acute ischemic stroke with vs without adjustment for stroke severity. JAMA 2012;308:257-64.

25 Smith EE, Shobha N, Dai D, et al. Risk score for in-hospital ischemic stroke mortality derived and validated within the Get With The Guidelines-Stroke Program. Circulation 2010:122:1496-504.

26 Quinn TJ, Dawson J, Lees JS, et al. Time spent at home poststroke: "home-time" a meaningful and robust outcome measure for stroke trials. Stroke 2008;39:231-3.

27 Elixhauser A, McCarthy EM. Clinical classifications for health policy research, version 2: Hospital inpatient statistics. Healthcare Cost and Utilization Project (HCUP 3) research note 1. Agency for Health Care Policy and Research, 1996. [AHCPR publication No 96-0017.]

28 Sacco RL, Kasner SE, Broderick JP, et al. An updated definition of stroke for the 21st century: a statement for healthcare professionals from the American Heart Association/American Stroke Association. Stroke 2013:44:2064-89.

29 Lichtman JH, Jones SB, Wang Y, et al. Outcomes after ischemic stroke for hospitals with and without Joint Commission-certified primary stroke centers. Neurology 2011;76:1976-82.

30 Wahl PM, Rodgers K, Schneeweiss S, et al. Validation of claims-based diagnostic and procedure codes for cardiovascular and gastrointestinal serious adverse events in a commercially-insured population. Pharmacoepidemiol Drug Saf2010:19:596-603.

31 Austin PC. Balance diagnostics for comparing the distribution of baseline covariates between treatment groups in propensity-score matched samples. Stat Med 2009;28:3083-107.

32 Curtis LH, Hammill BG, Eisenstein EL, et al. Using inverse probabilityweighted estimators in comparative effectiveness analyses with observational databases. Med Care 2007:45:S103-7.

33 Rosenbaum PR, Rubin DB. The central role of the propensity score in observational studies for causal effects. Biometrika 1983;70:41-55.

34 Hart RG, Pearce LA, Aguilar MI. Meta-analysis: antithrombotic therapy to prevent stroke in patients who have nonvalvular atrial fibrillation. Ann Intern Med 2007;146:857-67.

35 Bushnell CD, Zimmer LO, Pan W, et al. Persistence with stroke prevention medications 3 months after hospitalization. Arch Neurol 2010;67:1456-63.

36 McAlister FA. The end of the risk-treatment paradox? A rising tide lifts all boats. J Am Coll Cardiol 2011;58:1766-7.

37 Bungard TJ, Ghali WA, McAlister FA, et al. Physicians' perceptions of the benefits and risks of warfarin for patients with nonvalvular atrial fibrillation. CMAI 2001;165:301-2.

38 Mishra NK, Shuaib A, Lyden P, et al. Home time is extended in patients with ischemic stroke who receive thrombolytic therapy: a validation study of home time as an outcome measure. Stroke 2011;42:1046-50.

39 Graham DJ, Reichman ME, Wernecke M, et al. Cardiovascular, bleeding, and mortality risks in elderly Medicare patients treated with dabigatran or warfarin for nonvalvular atrial fibrillation. Circulation 2015;131:157-64. 